02

\title{
Фазовые переходы в гибридных SFS-структурах с тонкими сверхпроводящими слоями
}

\author{
(C) A.B. Самохвалов ${ }^{1,2}$ \\ ${ }^{1}$ Институт ффизики микроструктур РАН, \\ Нижний Новгород, Россия \\ ${ }^{2}$ Нижегородский госуниверситет им. Н.И. Лобачевского, \\ Нижний Новгород, Россия \\ E-mail: samokh@ipm.sci-nnov.ru
}

\begin{abstract}
На основе линеаризованных уравнений Узаделя выполнены расчеты критической тем пературы $T_{c}$ фазового перехода в сверхпроводящее состояние гибридной структуры сверх проводник/ферромагнетик/сверхпроводник $(S F S)$ с эффектом близости. Показано, что эффект близости между $S$ и $F$ металлами и обменное взаимодействие могут индуцировать неоднородное сверхпроводящее состояние с продольной слоям модуляцией сверхпроводящего параметра порядка $\Delta \propto \exp (i p z)$, которое характеризуется отличным от нуля значением волнового числа $p$, описывающего неустойчивость Ларкина-Овчинникова-Фульде-Феррелла. Изучено влияние этой неустойчивости на переходы между 0- и $\pi$-состояниями $S F S$-структуры. Показано, что $0-\pi$ переход сопровождается немонотонной зависимостью как критической температуры $T_{c}$, так и эффективной глубины проникновения магнитного поля $\Lambda$ в гибридную структуру от характерного размера ферромагнитной области.
\end{abstract}

Работа выполнялась при финансовой поддержке гранта РНФ № 151210020.

DOI: $10.21883 /$ FTT.2017.11.45048.07k

\section{1. Введение}

Необычное поведение гибридных структур, состоящих из чередующихся слоев сверхпроводника $(S)$ и ферромагнетика $(F)$ с эффектом близости $[1]$, как правило, связано с явлением $\pi$-сверхпроводимости [2], при котором устанавливается нетривиальная разность фаз $\Delta \theta=\pi$ между соседними сверхпроводящими слоями. Эффект близости на $S F$-границах и обменное взаимодействие приводят к появлению в ферромагнетике сверхпроводящих корреляций, амплитуда которых осциллирует и затухает в направлении, перпендикулярном $S F$-границе [3-5]. Частичное или полное подавление сверхпроводимости в $S$-слоях зависит от структуры парной волновой функции, т. е. от типа основного состояния $(0$ или $\pi)$, которое устанавливается в $S F S$-структуре. Фазовый переход между 0 - и $\pi$-состояниями гибридной структуры проявляется в немонотонной зависимости критической температуры перехода структуры в сверхпроводящее состояние $T_{c}$ от толщины $F$-слоя и экспериментально определяется по заметному подавлению джозефсоновского тока $S F S$-перехода $[3,4,6,7]$. Для определения критической температуры $T_{c}$-перехода гибридной $S F$-структуры в сверхпроводящее состояние используют измерения температурной зависимости сопротивления образца [8-10], его магнитной восприимчивости [11], или нелинейного СВЧ-отклика [12].

В последнее время ведутся активные исследования джозефсоновских переходов, в которых из-за различных особенностей туннелирования через барьер возникает неоднородное вдоль слоев распределение джозефсоновской разности фаз. В гибридных $S F S$-структурах это достигается путем изменения толщины ферромагнитного слоя [13] и/или при использовании комбинированного барьера, состоящего из участков нормального $(N)$ металла и ферромагнетика [14]. Подобные системы с искусственно созданным чередованием 0 и $\pi$ контактов обладают необычной (отличной от фраунгоферовой) зависимостью критического тока $I_{c}$ от внешнего магнитного поля $H_{0}[15,16]$ и допускают спонтанную генерацию вихрей с магнитным потоком, равным доле кванта магнитного потока $\Phi_{0}=\pi \hbar c /|e|$ на границах между 0 и $\pi$ участками перехода $[17,18]$. Джозефсоновская разность фаз в основном состоянии $0-\pi$ перехода может принимать произвольное значение $0<\varphi<\pi \quad[19,20]$, что позволяет использовать такой $\varphi$-контакт в качестве источника заданного фазового сдвига (фазовой батареи) в контурах сверхпроводящих квантовых интерферометров [21]. Предложенный в [14] дизайн гибридной структуры с комбинированным $F / N$-барьером может оказаться перспективным для разработки на его основе сверхпроводящей ячейки памяти. Заметим, что при отсутствии структурных неоднородностей на $F S$-границе продольная слоям фазовая модуляция сверхпроводящего параметра порядка возможна из-за формирования неоднородного состояния Ларкина-Овчинникова-Фульде-Феррелла (ЛОФФ) [22,23]. Влияние продольного ЛОФФ-состояния на свойства слоистых гибридных систем сверхпроводник/ферромагнетик было изучено недавно в работе [24], где была показана связь ЛОФФ-неустойчивости и аномального эффекта Мейснера в подобных структурах. При наличии сильного спин-орбитального взаимодействия в ферромагнетике продольная ЛОФФ-модуляция 
может сопровождаться возникновением спонтанных токов, локализованных вблизи $S F$-границы $[25,26]$.

Особенности эффекта близости на $S F$-границе приводят также к аномальному поведению экранирующих свойств гибридных структур: немонотонной зависимости эффективной длины проникновения магнитного поля в $S F$-бислой от толщины ферромагнетика [27-29], парамагнитному эффекту Мейснера [30-32], ухудшению экранирующих свойств $S F S$-структуры при понижении температуры $T$ [29,33,34]. В случае изучения экранирующих свойств гибридных SF структур в качестве исследуемой величины выступает эффективная глубина проникновения магнитного поля $\Lambda$, которая вблизи $T_{c}$ определяется средней по объему $S F$-структуры плотностью куперовских пар: $\Lambda^{-2} \sim\left(n^{s}-n^{t}\right)$, где индекс $s(t)$ относится к синглетной (триплетной) составляющей конденсата. Различное распределение компонент $n^{s}$ и $n^{t}$ в структуре может привести к изменению знака $\Lambda^{-2}$, что и служит причиной ЛОФФ-неустойчивости в плоскости слоев [24].

В данной работе изучены экранирующие свойства слоистой $S F S$-структуры с диффузным типом проводимости при температуре, близкой к температуре фазового перехода в сверхпроводящее состояние. Получены условия появления дополнительной ЛОФФ-модуляции в плоскости слоев, которая подавляет парамагнитный эффект Мейснера в такой системе. В разд. 2 обсуждается используемая модель и приведены основные уравнения для ее описания. В разд. 3 на основе линеаризованных уравнений Узаделя получены аналитические решения, описывающие неоднородное сверхпроводящее состояние $(0$ или $\pi)$ с произвольным значением волнового числа $p$ в плоскости слоев, возникающее под действием обменного поля и эффекта близости. Выполнены расчеты критической температуры сверхпроводящего перехода $T_{c}$ и изучены переключения между 0 - и $\pi$-состояниями с различными значениями волнового числа $p$, которые сопровождаются немонотонной зависимостью $T_{c}$ от размера ферромагнитной области. В разд. 4 выполнены расчеты эффективной глубины проникновения магнитного поля $\Lambda$ в $S F S$-структуру и изучено влияние ЛОФФнеустойчивости на экранирующие свойства гибридной структуры. В заключении приведены основные результаты работы.

\section{2. Модель и основные уравнения}

Рассмотрим модельную трехслойную $S F S$-структуру с тонкими сверхпроводящими слоями с толщиной $d_{s}$ порядка сверхпроводящей длины когерентности $\xi_{s}=\sqrt{\hbar D_{s} / 2 \pi T_{s c}}$ и ферромагнитным барьером толщиной $d_{f}$. Будем считать, что образующие гибридную структуру $S$ - и $F$-металлы с коэффициентами диффузии $D_{s, f}$ соответственно удовлетворяют условиям „грязного“ предела [35], так что длина свободного пробега электронов в металлах $l=v_{F} \tau$ достаточно мала $\left(l \ll \xi_{0}\right)$, а критическая температура сверхпроводника $T_{c s}$ (при отсутствии эффекта близости и/или внешнего магнитного поля) и энергия обменного поля ферромагнетика $h$ удовлетворяют условиям $T_{c s} \tau \ll 1$ и $h \tau \ll 1$. Здесь $\tau$-характерное время упругого рассеяния электронов на немагнитных примесях, а $\xi_{0}=\hbar v_{F} / \Delta_{0}-$ длина когерентности при $T=0$.

Используя стандартную параметризацию квазиклассической аномальной функции Грина $[36,37]$

$$
\hat{f}_{s, f}=f_{s, f}^{s}+\mathbf{f}_{s f}^{t} \hat{\sigma},
$$

можно записать линеаризованные уравнения Узаделя [38] для компонент аномальной функции Грина $f_{f}^{s, t}$ в ферромагнетике с нулевым потенциалом спаривания в виде (подробнее, см. обзоры [39-41])

$$
\begin{gathered}
-\left(D_{f} / 2\right) \nabla^{2} f_{f}^{s, t}+\left|\omega_{n}\right| f_{f}^{s, t}+i h \operatorname{sgn} \omega_{n} f_{f}^{t, s}=0, \\
-\left(D_{s} / 2\right) \nabla^{2} f_{s}^{s}+\left|\omega_{n}\right| f_{s}^{s}=\Delta(\mathbf{r}), \\
-\left(D_{s} / 2\right) \nabla^{2} f_{s}^{t}+\left|\omega_{n}\right| f_{s}^{t}=0 .
\end{gathered}
$$

Здесь $\omega_{n}=(2 n+1) \pi T_{c}$ - мацубаровские частоты для температуры $T_{c}, f_{s}^{s}\left(f_{f}^{s}\right)$ - амплитуда синглетной компоненты, вектор $\mathbf{f}_{s}^{t}=f_{s}^{\prime} \mathbf{z}_{0}\left(\mathbf{f}_{f}^{t}=f_{f}^{t} \mathbf{z}_{0}\right)$ в случае однородного обменного поля и намагниченности $\mathbf{M}=M \mathbf{z}_{0}$ описывает триплетную компоненту в сверхпроводнике (ферромагнетике), а $\hat{\sigma}=\left(\sigma_{x}, \sigma_{y}, \sigma_{z}\right)-$ вектор матриц Паули. Триплетная компонента $f_{s, f}^{t}$ описывает сверхпроводящие корреляции с нулевой проекцией суммарного спина на ось квантования, которые затухают в ферромагнетике на том же масштабе $\xi_{f}=\sqrt{\hbar D_{f} / h}$, что и синглетные корреляции $f_{f}^{s}$. Параметр порядка $\Delta(\mathbf{r})$ отличен от нуля только в $S$ слоях и удовлетворяет уравнению самосогласования

$$
\Delta(\mathbf{r})=\pi T \rho \sum_{\omega_{n}} f_{s}^{s}\left(\mathbf{r}, \omega_{n}\right),
$$

в котором ответственная за синглетное спаривание константа $\rho$ постоянна в сверх проводнике и равна нулю в ферромагнетике. Уравнения Узаделя (2), (3), (4) следует дополнить граничными условиями Куприянова-Лукичева [42] на $F S$ границах $\left(x= \pm x_{f}=d_{f} / 2\right)$ для синглетной и триплетной компонент

$$
\begin{gathered}
\sigma_{s} \partial_{x} f_{s}^{s, t}=\sigma_{f} \partial_{x} f_{f}^{s, t}, \\
f_{s}^{s, t}=f_{f}^{s, t} \pm \gamma_{b} \xi_{n} \partial_{x} f_{f}^{s, t},
\end{gathered}
$$

а также свободными граничными условиями на внешних поверхностях структуры $\left(x= \pm x_{s}= \pm\left(d_{f} / 2+d_{s}\right)\right)$

$$
\partial_{x} f_{s}^{s, t}=0 \text {. }
$$

Здесь $\sigma_{s}$ и $\sigma_{f}-$ удельные проводимости $S$ - и $F$-металлов в нормальном состоянии, параметр $\gamma_{b}$ характеризует прозрачность $S F$-границы и определяется сопротивлением границы на единицу площади $R b$ $\left(\gamma_{b} \xi_{s}=R_{b} \sigma_{f}\right), \partial_{x}$ обозначает производную в направлении $x$, а $\xi_{n}=\sqrt{\hbar D_{f} / 2 \pi T_{c s}}$ - длина когерентности в несверхпроводящем ферромагнетике. 

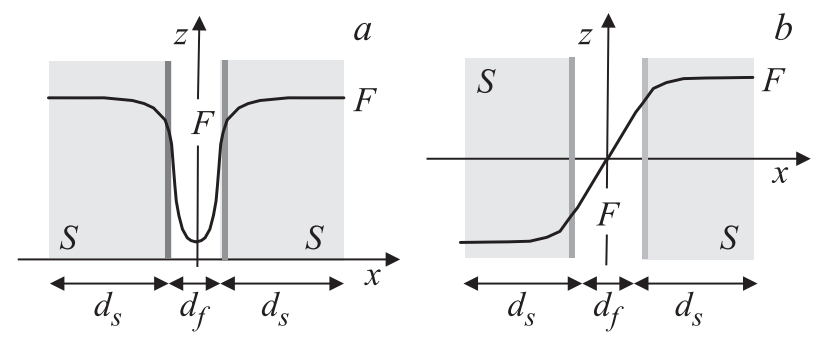

Рис. 1. Качественный вид распределения волновой функции куперовских пар $F$ в трехслойной $S F S$-структуре: $(a)$ фаза $[F(-x)=F(x)] ;(b) \pi$-фаза $[F(-x)=-F(x)]$.

\section{3. Неоднородные сверхпроводящие состояния в трехслойной SFS-структуре}

Получим аналитические решения, описывающие пространственное распределение синглетной и триплетной компонент аномальной функции Грина в гибридной структуре, изображенной на рис. 1 для 0 - и $\pi$-состояний. Будем искать решения уравнений $(2)$ в $F$ слое $\left(|x| \leq x_{f}\right)$ с заданным волновым числом $p$ в виде

$$
f_{f}^{s, t}\left(\mathbf{r}, \omega_{n}\right)=F_{f}^{s, t}\left(x, \omega_{n}\right) \exp (i p z),
$$

учитывая тем самым возможность ЛОФФ-модуляции с пространственным масштабом $2 \pi / p$, при которой фаза сверхпроводящего параметра порядка $\Delta$ осциллирует вдоль $S F$-границы. Распределение комплексной амплитуды в поперечном слоям направлении (вдоль оси $x$ )

$$
F_{f}^{s, t}\left(x, \omega_{n}\right)=C_{f}^{s, t}\left(\omega_{n}\right) \chi^{0, \pi}\left(q_{p} x\right)
$$

отличается для -0 и $\pi$-состояний с учетом симметрии решения

$$
\chi^{0}(u)=\operatorname{ch} u, \quad \chi^{\pi}(u)=\operatorname{sh} u .
$$

Для решений вида (9), (10) уравнение (2) при $h \gg \pi T_{c s}$ сводится к линейной алгебраической системе

$$
-\xi_{f}^{2}\left(q_{p}^{2}-p^{2}\right) C_{f}^{s, t}+2 i \operatorname{sgn} \omega_{n} C^{t, s}=0
$$

относительно комплексных коэффициентов $C_{f}^{s, t}\left(\omega_{n}\right)$, зависящих от мацубаровской частоты $\omega_{n}$. Система $(12)$ имеет нетривиальное решение, если

$$
q_{p}^{2}=\left(q_{p 1}+i q_{p 2}\right)^{2}=p^{2} \pm 2 i / \xi_{f}^{2} .
$$

Дисперсионное уравнение (13) устанавливает простое соотношение между коэффициентами $C_{f}^{s, t}\left(\omega_{n}\right)$

$$
C_{f}^{t}\left(\omega_{n}\right)= \pm \operatorname{sgn} \omega_{n} C_{f}^{s}\left(\omega_{n}\right)
$$

Учитывая соотношение (14) между коэффициентами $C_{f}^{s, t}\left(\omega_{n}\right)$, можно записать выражения для амплитуд синглетной $F_{f}^{s}\left(x, \omega_{n}\right)$ и триплетной $F_{s}^{t}\left(x, \omega_{n}\right)$ компонент в $F$-слое $\left(|x| \leq x_{d}\right)$ для заданного значения волнового числа $p$ в виде

$$
\begin{gathered}
F_{f}^{s}\left(x, \omega_{n}\right)=A_{n} \chi^{0, \pi}\left(q_{p} x\right)+\tilde{A}_{n} \chi^{0, \pi}\left(q_{p}^{*} x\right), \\
F_{f}^{t}\left(x, \omega_{n}\right)=\operatorname{sgn} \omega_{n}\left[A_{n} \chi^{0, \pi}\left(q_{p} x\right)-\tilde{A}_{n} \chi^{0, \pi}\left(q_{p}^{*} x\right)\right]
\end{gathered}
$$

для 0 - и $\pi$-состояний $S F S$-структуры.

Предполагая сверхпроводящий слой тонким $\left(d_{s} \lesssim \xi_{s}\right)$ проинтегрируем уравнения (3), (4) по толщине $S$-слоя, используя граничное условие (8) и пренебрегая изменением функций $f_{s}^{s, t}$ и $\Delta$ в поперечном $x$ направлении $[39,43]$

$$
f_{s}^{s, t}\left(\mathbf{r}, \omega_{n}\right) \simeq F_{s}^{s, t}\left(\omega_{n}\right) \exp (i p z), \quad \Delta(\mathbf{r}) \simeq \Delta_{s} \exp (i p z) .
$$

В результате получим следующее соотношение между амплитудой $F_{s}^{s, t}$ и производной $\partial_{x} F_{s}^{s, t}$ компонент аномальной функции Грина на $F S$-границе $\left(x=x_{f}\right)$

$$
\partial_{x} F_{s}^{s}=\frac{d_{s}}{\pi T_{c s} \xi_{s}^{2}}\left[\Delta_{s}-\Omega_{n} F_{s}^{s}\right], \partial_{x} F_{s}^{t}=-\frac{d_{s} \Omega_{n}}{\pi T_{c s} \xi_{s}^{2}} F_{s}^{t},
$$

где $\Omega_{n}=\left|\omega_{n}\right|+D_{s} p^{2} / 2$.

Подставляя решения (15),(16) в граничные условия (6), (7) и (18) на $F S$-границе получим, что для действительного значения параметра порядка $\Delta_{s}$ синглетная $f_{s}^{s}$ и триплетная $f_{s}^{t}$ компоненты аномальной функции Грина в сверхпроводнике и ферромагнетике определяются следующими выражениями

$$
\begin{gathered}
F_{s}^{s}\left(\omega_{n}\right)=\operatorname{Re}\left[A_{n s}\right], \quad F_{s}^{t}\left(\omega_{n}\right)=i \operatorname{sgn} \omega_{n} \operatorname{Im}\left[A_{n s}\right] \\
F_{f}^{s}\left(r, \omega_{n}\right)=\operatorname{Re}\left[A_{n f} \chi^{0, \pi}\left(q_{p} x\right)\right], \\
F_{f}^{t}\left(r, \omega_{n}\right)=i \operatorname{sgn} \omega_{n} \operatorname{Im}\left[A_{n f} \chi^{0, \pi}\left(q_{p} x\right)\right],
\end{gathered}
$$

в которых комплексные амплитуды

$$
\begin{gathered}
A_{n s}=\frac{\Delta_{s}}{\left|\omega_{n}\right|+\Omega^{0, \pi}(p)}, \\
A_{n, f}=\frac{A_{n s}}{\chi^{0, \pi}\left(u_{f}\right)+\gamma_{b} \xi_{n} q_{p} \chi^{\pi, 0}\left(u_{f}\right)},
\end{gathered}
$$

$\left(u_{f}=q_{p} x_{f}\right)$ соответствующие мацубаровской частоте $\omega_{n}$ зависят от параметра распаривания

$$
\Omega^{0, \pi}=\pi T_{c s} \xi_{s}^{2}\left[p^{2}+\frac{\sigma_{f} / \sigma_{s}}{d_{s}\left(\gamma_{0} \xi_{n}+\chi^{0, \pi}\left(u_{f}\right) / q_{p} \chi^{\pi, 0}\left(u_{f}\right)\right)}\right] .
$$

Легко видеть, что амплитуды синглетных компонент аномальной функции Грина $F_{s}^{s}$ и $F_{f}^{s}$ оказываются действительными четными функциями мацубаровских частот $\left(F_{s, f}^{s}\left(r,-\omega_{n}\right)=F_{s, f}^{s}\left(r, \omega_{n}\right)\right)$. В то время как амплитуды триплетных компонент $F_{s, f}^{t}$ - чисто мнимые и нечетные функции $F_{s, f}^{t}\left(r,-\omega_{n}\right)=-F_{s, f}^{t}\left(r, \omega_{n}\right)[36,40,43]$.

Критическая температура сверхпроводящего перехода определяется уравнением самосогласования (5) для 

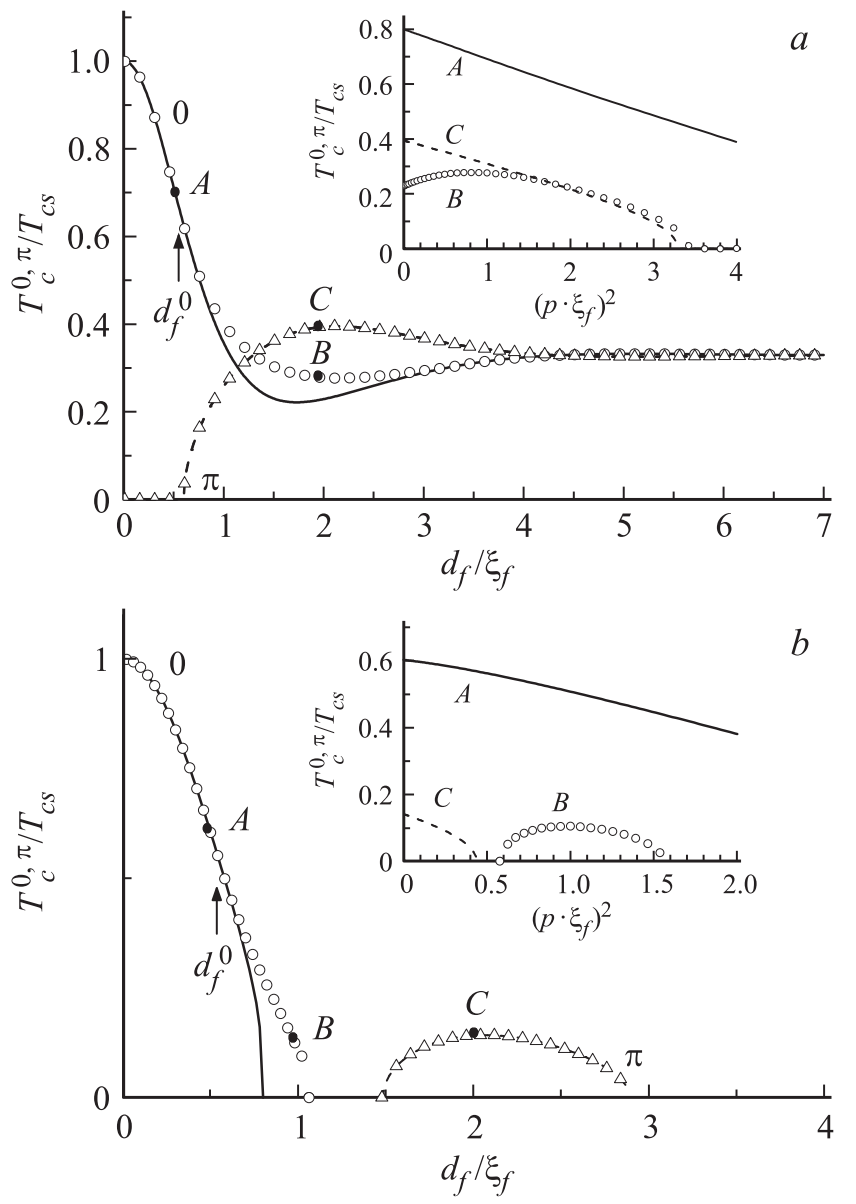

Рис. 2. Зависимость критической температуры $T_{c}^{0, \pi}(0)$ (линии) и $T_{c}^{0, \pi}\left(p_{m}\right)$ (символы) от толщины $F$-слоя $d_{f}$ для 0(сплошная линия) и $\pi$ - (пунктирная линия) состояний: $a-$ $d_{s}=0.5 \xi_{s}, b-d_{s}=0.4 \xi_{s}$. Остальные параметры: $\sigma_{s} / \sigma_{f}=0.5$, $\xi_{s} / \xi_{f}=0.1, \xi_{n} / \xi_{f}=1.5, \gamma_{b}=0.75$. На вставке показаны типичные зависимости критической температуры $T_{c}^{0, \pi}(p)$ от волнового числа $p$ продольной ЛОФФ-модуляции для точек $A-C$ на зависимости $T_{c}^{0, \pi}$ от толщины $F$-слоя $d_{f}$.

синглетного параметра порядка $\Delta(\mathbf{r})$, которое с учетом решений (19), (20) можно записать в традиционном виде

$$
\ln \frac{T_{c}^{0, \pi}(p)}{T_{c s}}=\Psi\left(\frac{1}{2}\right)-\operatorname{Re}\left[\Psi\left(\frac{1}{2}+\frac{\Omega^{0, \pi}(p)}{2 \pi T_{c}^{0, \pi}(p)}\right)\right],
$$

где $\Psi$ - дигамма функция. Параметр $\Omega^{0, \pi}(p)(21)$ учитывает орбитальный и парамагнитный механизмы распаривания, совместное действие которых приводит к формированию неоднородных 0 - и $\pi$-сверхпроводящих состояний с ЛОФФ-модуляцией вдоль слоев. Критическая температура сверхпроводящего перехода $T_{c}$ определяется, как обычно, максимальным значением $T_{c}^{0, \pi}(p)$

$$
T_{c}=\max _{p}\left\{T_{c}^{0}(p), T_{c}^{\pi}(p)\right\}
$$

и достигается при значении волнового числа $p=p_{m}$, которое в общем случае может быть отличным от нуля.
На рис. 2 показаны типичные зависимости температуры перехода $T_{c}^{0, \pi}(22), \quad(23)$ в сверхпроводящее 0- или $\pi$-состояние от толщины слоя ферромагнетика $d_{f}$ без учета $(p=0)$ и с учетом $(p \neq 0)$ продольной ЛОФФмодуляции для двух близких значений отношения $\sigma_{s} / \sigma_{f}$. Как видно из рисунка, для тонких $F$-барьеров с толщиной $d_{f} \ll \xi_{f}$ устанавливается однородное по $z$ сверхпроводящее состояние $\left(p_{m}=0\right)$, у которого разность фаз параметров порядка $\Delta \theta$ в сверхпроводящих слоях отсутствует (0-состояние), эффект близости слабый, и критическая температура этого состояния близка к критической температуре объемного сверхпроводника $T_{c s}$. C увеличением толщины $F$-слоя влияние обменного поля ферромагнетика растет, а критическая температуpa $S F S$-структуры уменьшается, оставаясь по-прежнему максимальной при $p_{m}=0$ (кривая $A$ на вставке к рис. 2). При $d_{f}>d_{f}^{0}$ зависимость $T_{c}(p)$ качественно меняется: максимальное значение критической температуры достигается при $p=p_{m} \neq 0$ (кривая $B$ на вставке к рис. 2), и $S F$-структура переходит модулированное ЛОФФ-0-состояние $\left\{0, p_{m}\right\}$. На рис. 3 показана зависимость оптимального волнового числа $p_{m}$ от толщины $F$ слоя $d_{f}$ для 0- и $\pi$-состояний. При дальнейшем увеличении $d_{f}$ критическая температура $T_{c}^{0}\left(p_{m}\right)$ 0-состояния с ЛОФФ-модуляцией снижается. Одновременно начинают играть роль знакопеременные осцилляции парной волновой функции в ферромагнетике в поперечном направлении, поскольку толщина $d_{f}$ становится соизмеримой с периодом осцилляций $\sim \xi_{f 2}=1 / q_{p 2}$ аномальной функции Грина $F_{f}^{s, t}$. Это приводит к формированию однородного по $z \pi$-состояния $\{\pi, 0\}$, у которого разность фаз параметров порядка $\Delta \theta=\pi$, которое затем сменяется модулированным $\pi$-состоянием $\left\{\pi, p_{m}\right\}$. Таким образом,

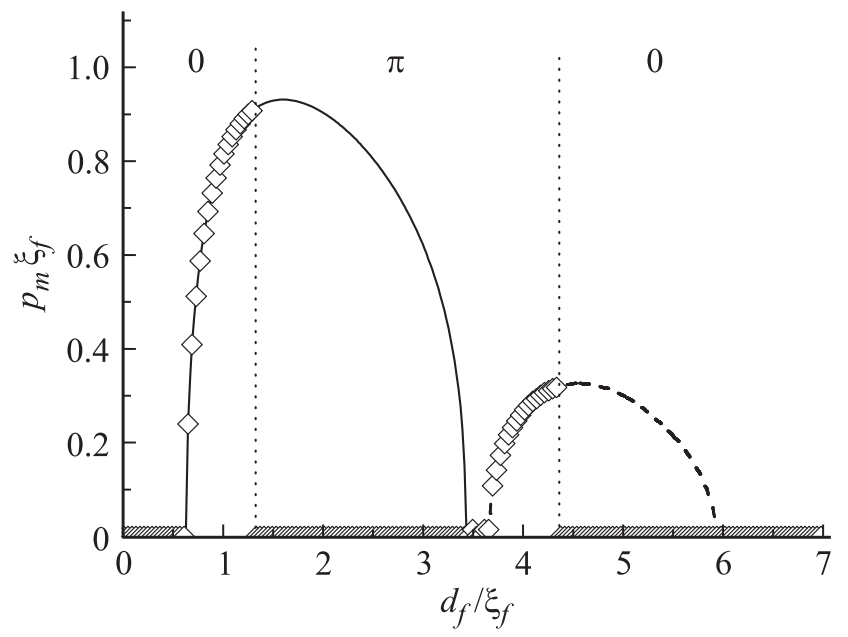

Рис. 3. Зависимость оптимального волнового числа $p_{m}$ от толщины $F$-слоя $d_{f}$ для 0 - (сплошная линия) и $\pi$ - (пунктирная линия) состояний. Символами показана зависимость $p_{m}\left(d_{f}\right)$, построенная с учетом условия (23). Области, соответствующие 0- и $\pi$-состояниям, разделены вертикальными точечными линиями. Параметры SFS-структуры совпадают с используемыми на рис. 2, $a$. 
эффект близости и обменное взаимодействие могут индуцировать в $S F S$-структуре как необычное $\pi$-состояние, так и продольную слоям ЛОФФ-модуляцию [24].

Как и в случае $S F$-бислоя продольная модуляция параметра порядка возникает в трехслойных $S F S$-структурах, у которых сверхпроводимость существенно ослаблена эффектом близости с ферромагнитным металлом, т.е. при $T_{c} \ll T_{c s}$. Поэтому для возникновения ЛОФФмодуляции сверхпроводящие слои должны быть достаточно тонкими $\left(d_{s}<\xi_{s}\right)$, а ферромагнитный металл низкоомным $\left(\sigma_{s}<\sigma_{f}\right)$. В этих условиях однородное по $z$ сверхпроводящее состояние может оказаться полностью подавленным $\left(T_{c}(0)=0\right)[43,44]$. Однако формирование неоднородного вдоль слоев $S$ состояния „расширяет“ область допустимых параметров $S F S$-структуры, при которых сверхпроводимость тем не менее существует $\left(T_{c}(p \neq 0)>0\right.$ ) (см. рис. 2, $\left.b\right)$. Поскольку необходимое для формирования ЛОФФ-состояния условие $T_{c} \ll T_{c, s}$, как правило, выполняется в окрестности $0-\pi$ перехода, возникновение продольной слоям фазовой модуляции приводит к заметному смещению положения перехода и может оказать влияние на характеристики джозефсоновской связи вблизи перехода $S F S$-структуры между 0и $\pi$-состояниями.

\section{4. Эффективная глубина проникновения магнитного поля}

Экранирующие свойства гибридных $S F$-структур при температурах $T$, близких к критической $T_{c}$ характеризуются эффективной глубиной проникновения магнитного поля $\Lambda$, зависящей от средней по объему плотности куперовских пар в структуре $n_{s}=\left\langle n_{s}(x)\right\rangle$, где $n_{s}(x) \propto \lambda^{-2}(x)$

$$
\Lambda^{-2}=\frac{2}{d} \int_{0}^{x_{s}} \frac{d x}{\lambda^{2}(x)} \propto n_{s} .
$$

Здесь $d=d_{d}+2 d_{s}$ - толщина $S F S$-структуры, а $\lambda(x)-$ лондоновская глубина проникновения, которая входит в соотношение

$$
\mathbf{j}=\frac{c}{4 \pi \lambda^{2}}\left(\frac{\hbar c}{2 e} \nabla \theta-\mathbf{A}\right)
$$

между экранирующим током $\mathbf{j}$ и векторным потенциалом $\mathbf{A}$ и следующим образом может быть записана через амплитуды $F_{f, s}^{s}$ и $F_{f, s}^{t}(19),(20)$ квазиклассических функций Грина (9), (10)

$$
\lambda_{s, f}^{-2}=\frac{8 \pi^{2} T_{c} \sigma_{s, f}}{\hbar c^{2}} \sum_{\omega_{n}} \operatorname{Re}\left[F_{s, f}^{2}\right]
$$

где $F_{s ; f}=F_{s, f}^{s}+F_{s, f}^{t}$ для $S$ - и $F$-слоев соответственно. Подставляя в $(24),(26)$ в качестве амплитуд квазиклассических функций Грина $F_{f, s}^{s}$ - и $F_{f, s}^{t}$-решения линеари-

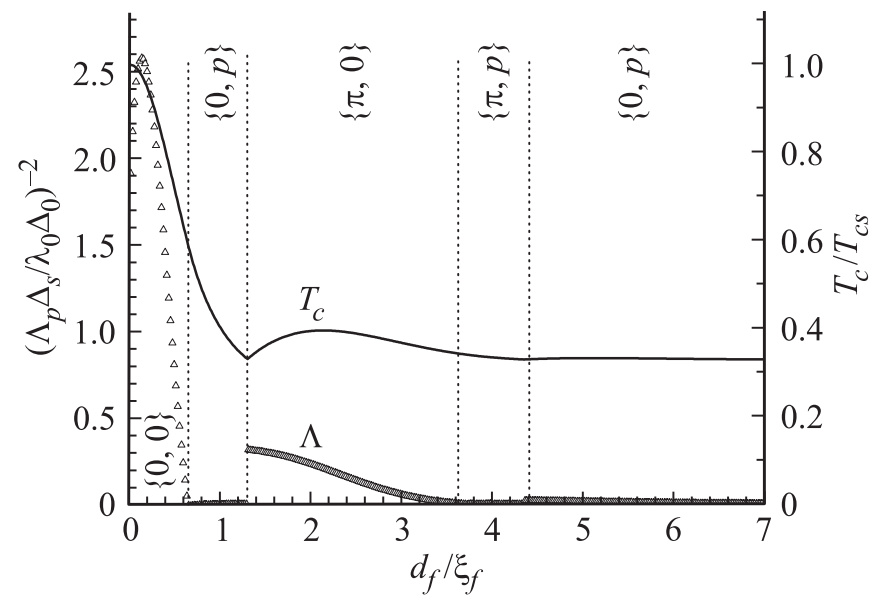

Рис. 4. Зависимость критической температуры $T_{c}(23)$ (сплошная линия), эффективной глубины проникновения $\Lambda(24)$ (символы) от радиуса $F$-слоя $d_{f}$. Глубина проникновения $\Lambda$ нормирована на величину $\lambda_{0}$, где $\lambda_{0}^{-2}=4 \pi^{2} \sigma_{s} \Delta_{0} / \hbar c^{2}$, a $\Delta_{0}=3.52 T_{c s}$. Области, соответствующие 0 - и $\pi$-состояниям, разделены вертикальными точечными линиями. Параметры $S F S$-структуры совпадают с используемыми на рис. $2, b$.

зованных уравнений Узаделя (19), (20), получим следующее выражение для эффективной глубины проникновения магнитного поля $\Lambda_{0, \pi}(p)$ для состояний $\{0, p\}$ и $\{\pi, p\}$ вида $(10),(17)$ соответственно

$$
\begin{aligned}
\Lambda_{0, \pi}^{-2}(p)= & \frac{8 \Delta_{s}^{2} \sigma_{s}}{\hbar c^{2} T_{c}^{0, \pi}} \operatorname{Re}\left\{\Psi_{1}\left(\frac{1}{2}+\frac{\Omega^{0, \pi}(p)}{2 \pi T_{c}^{0, \pi}}\right)\right. \\
& \left.\times\left[\frac{d_{s}}{d}+\frac{\sigma_{f} / \sigma_{s}\left(\text { th } u_{f} \pm 2 u_{f} / \mathrm{ch}^{2} u_{f}\right)}{4 q_{p} d Z_{0}^{2}}\right]\right\}, \\
Z_{0}=1 & +\gamma_{b} q_{p} \xi_{n} \text { th } u_{f}, \quad Z_{\pi}=\text { th } u_{f}+\gamma_{b} q_{p} \xi_{n},
\end{aligned}
$$

где $\Psi_{1}$ - тригамма функция. На линии фазового перехода второго рода при $T=T_{c}$ сверхпроводимость пропадает $\left(\Delta_{s}=0\right)$, а эффективная глубина проникновения (24) расходится $\left(\Lambda_{0, \pi}(p) \rightarrow \infty\right)$. При $T \lesssim T_{c}$ структура сверхпроводящего параметра порядка, соответствующая минимуму свободной энергии, может отличаться от (17) [45], и для определения равновесного значения $\Delta_{s}$ необходимо отказаться от линейного приближения (2)-(4) и использовать полные нелинейные уравнение Узаделя. Поэтому выражение (27) устанавливает лишь соотношение между сверхпроводящим параметром порядка $\Delta_{s}$ и эффективной глубиной проникновения магнитного поля $\Lambda_{0, \pi}(p)$ в $S F S$-структуру для состояний $\{0, p\}$ и $\{\pi, p\}$ при температуре $T$ близкой к критической $T_{c}$. На рис. 4 показаны зависимости критической температуры $T_{c}(23)$ и величины $\left(\Lambda \Delta_{s}\right)^{-2}=\left(\Lambda_{0, \pi}\left(p_{m}\right) \Delta_{s}\right)^{-2}(27)$ от толщины $F$-слоя $d_{f}$. Смена режимов происходит в соответствии с условием (23), при выполнении которого устанавливается состояние $\left\{0, p_{m}\right\}$ или $\left\{\pi, p_{m}\right\}$ с максимальной критической температурой фазового перехода. Заметим, 
что $0-\pi$ переходу, как правило, предшествует формирование в $S F S$-структуре состояния с продольной ЛОФФ-модуляцией, так что при 0-л переходе изменяется не только поперечная, но и продольная слоям структура парной волновой функции. Скачки величины $\left(\Lambda_{0, \pi}(p) \Delta_{s}\right)^{-2}$ при 0-л переходе связаны с качественными изменениями поперечной структуры (четности) парной волновой функции в $F$-слое. Подобные проявления $0 \rightarrow \pi$ перехода в трехслойных $S F S$-структурах наблюдались в экспериментах [29,33] по заметному уменьшению средней плотности куперовских пар $n_{s}$ и увеличению эффективной глубины проникновения $\Lambda \propto 1 / \sqrt{n_{s}}$ при понижении температуры $T$.

Условие $\Lambda_{0, \pi}^{-2}(0)=0$, при котором пропадает совокупный диамагнитный отклик структуры, определяет границу появления ЛОФФ-неустойчивости однородного по $z$ состояния (0 или $\pi)$ в SFS-структуре. Как и в случае $S F$-бислоя [24], этот критерий глобальной устойчивости сверхпроводящего $0(\pi)$ состояния удобно записать в виде

$$
\begin{aligned}
\Lambda_{0, \pi}^{-2}(0)= & -\frac{16 \Delta_{s}^{2} \sigma_{s} d_{s}}{\hbar c^{2} T_{c}^{0, \pi} T_{c s} d \xi_{s}^{2}}\left[1-\operatorname{Re}\left\{\frac{\Omega^{0, \pi}(0)}{2 \pi T_{c}^{0, \pi}}\right.\right. \\
& \left.\left.\times \Psi_{1}\left(\frac{1}{2}+\frac{\Omega^{0, \pi}(0)}{2 \pi T_{c}^{0, \pi}}\right)\right\}\right]\left.\frac{d T_{c}^{0, \pi}}{d p^{2}}\right|_{p=0},
\end{aligned}
$$

используя решение $T_{c}^{0, \pi}(p)$ уравнения самосогласования (22). Таким образом, если $\Lambda_{0, \pi}^{-2}(0) \geq 0$ $\left(d T_{c}^{0, \pi} / d p^{2} \leq 0\right.$ при $\left.p=0\right)$, то в $S F S$-структуре устанавливается однородное по $z$ состояние $(0$ или $\pi)$, для которого критическая температура $T_{c}^{0, \pi}$ максимальна. Если $\Lambda_{0, \pi}^{-2}(0)\left(d T_{c}^{0, \pi} / d p^{2}>0\right.$ при $\left.p=0\right)$, то максимальная критическая температура $T_{c}(23)$ достигается при $p=p_{m} \neq 0$, когда $T_{c}^{0, \pi}(p)$ принимает максимальное значение $\left(d T_{c}^{0, \pi} / d p^{2}=0\right.$ при $\left.p=p_{m}\right)$. При этом следует ожидать восстановление диамагнитного отклика гибридной структуры [24].

\section{5. Заключение}

Таким образом, в данной работе приведены результаты теоретических исследований неоднородных состояний в гибридных слоистых структурах сверхпроводник/ферромагнетик/сверхпроводник с эффектом близости. Показано, что обменное взаимодействие и эффект близости могут вызвать как переходы между сверхпроводящими 0- и $\pi$-состояниями, так и формировать неоднородное состояние типа Ларкина-Овчинникова-Фульде-Феррелла с фазовой модуляцией вдоль слоев на масштабе $2 \pi / p$, определяемом волновым числом $p \approx 1 / \xi_{f}$. На основе линеаризованных уравнений Узаделя выполнены расчеты критической температуры фазового перехода $T_{c}$ в неоднородные 0 - и $\pi$-состояния с различными значениями $p$, которая немонотонно зависит от толщины слоя ферромагнитнетика $d_{f}$. Изучены особенности, устойчивость и условия формирования продольного слоям ЛОФФ-состояния и проанализированы экранирующие свойства гибридной $S F S$-структуры. Показано, что $0-\pi$ переход сопровождается развитием (подавлением) ЛОФФ-неустойчивости. Фазовый переход в ЛОФФ-состояние совпадает с подавлением диамагнитного отклика, так что эффективная глубина проникновения магнитного поля $\Lambda$ в $S F S$-структуру расходится $\left(\Lambda^{-2}=0\right)$. Переключения между 0 - и $\pi$ - и/или ЛОФФсостояниями сопровождается немонотонной зависимостью $\Lambda$ от толщины ферромагнитного слоя $d_{f}$.

\section{Список литературы}

[1] R. Holm, W. Meissner. Z. Physik 74, 715 (1932).

[2] Л.Н. Булаевский, В.В. Кузий, А.А. Собянин. Письма в ЖЭТФ 25, 314 (1977)

[3] А.И. Буздин, Л.Н. Булаевский, С.В. Панюков. Письма в ЖЭТФ 35, 147 (1982).

[4] А.И. Буздин, М.Ю. Куприянов. Письма в ЖЭТФ 53, 308 (1991).

[5] А.И. Буздин, В. Вуйчич, М.Ю. Куприянов. ЖЭТФ 101, 231 (1992).

[6] V.V. Ryazanov, V.A. Oboznov, A.Yu. Rusanov, A.V. Veretennikov, A.A. Golubov. J. Aarts. Phys. Rev. Lett. 86, 2427 (2001).

[7] T. Kontos, M. Aprili, J. Lesueur, F. Genet, B. Stephanidis, R. Boursier. Phys. Rev. Lett. 89, 137007 (2002).

[8] J.S. Jiang, D. Davidović, D.H. Reich, C.L. Chien. Phys. Rev. Lett. 74, 314 (1995).

[9] I.A. Garifullin, D.A. Tikhonov, N.N. Garif'yanov, L. Lazar, Yu.V. Goryunov, S.Ya. Khlebnikov, L.R. Tagirov, K. Westerholt, H. Zabel. Phys. Rev. B 66, 020505 (2002).

[10] V.I. Zdravkov, J. Kehrle, G. Obermeier, S. Gsell, M. Schreck, C. Müller, H.A. Krug von Nidda, J. Lindner, J. MoosburgerWill, E. Nold, R. Morari, V.V. Ryazanov, A.S. Sidorenko, S. Horn, R. Tidecks, L.R. Tagirov. Phys. Rev. B 82, 054517 (2010).

[11] Th. Mühge, N.N. Garif'yanov, Yu.V. Goryunov, G.G. Khaliullin, L.R. Tagirov, K. Westerholt, I.A. Garifullin, H. Zabel. Phys. Rev. Lett. 77, 1857 (1996).

[12] С.Н. Вдовичев, Ю.Н. Ноздрин, Е.Е. Пестов, П.А. Юнин, А.В. Самохвалов. Письма в ЖЭТФ 104, 336 (2016).

[13] M. Weides, M. Kemmler, E. Goldobin, H. Kohlstedt, R. Waser, D. Koelle, R. Kleiner. Phys. Rev. Lett. 97, 247001 (2006).

[14] S.V. Bakurskiy, N.V. Klenov, I.I. Soloviev, M.Yu. Kupriyanov, A.A. Golubov. Appl. Phys. Lett. 108, 042602 (2016).

[15] H. Hilgenkamp, J. Mannhart, B. Mayer. Phys. Rev. B 53, 14586 (1996).

[16] C. Gürlich, S. Scharinger, M. Weides, H. Kohlstedt, R.G. Mints, E. Goldobin, D. Koelle, R. Kleiner. Phys. Rev. B 81, 094502 (2010).

[17] L.N. Bulaevskii, V.V. Kuzii, A.A. Sobyanin. Solid State Commun. 25, 1053 (1978).

[18] R.G. Mints. Phys. Rev. B 57, R3221 (1998).

[19] A. Buzdin, A.E. Koshelev. Phys. Rev. B 67, 220504 (2003).

[20] H. Sickinger, A. Lipman, M. Weides, R.G. Mints, H. Kohlstedt, D. Koelle, R. Kleiner, E. Goldobin. Phys. Rev. Lett. 109, 107002 (2012). 
[21] A.K. Feofanov, V.A. Oboznov, V.V. Bol'ginov, J. Lisenfeld, S. Poletto, V.V. Ryazanov, A.N. Rossolenko, M. Khabipov, D. Balashov, A.B. Zorin, P.N. Dmitriev, V.P. Koshelets, A.V. Ustinov. Nature Phys. 6, 593 (2010).

[22] А.И. Ларкин, Ю.Н. Овчинников. ЖЭТФ 47, 1136 (1964).

[23] P. Fulde, R.A. Ferrell. Phys. Rev. 135, A550 (1964).

[24] S. Mironov, A. Mel'nikov, A. Buzdin. Phys. Rev. Lett. 109, 237002 (2012).

[25] I.V. Bobkova, Yu.S. Barash. Письма в ЖЭТФ 80, 563 (2004).

[26] S. Mironov, A. Buzdin. Phys. Rev. Lett. 118, 077001 (2017).

[27] T.R. Lemberger, I. Hetel, A.J. Hauser, F.Y. Yang. J. Appl. Phys. 103, 07C701 (2008).

[28] M. Houzet, J.S. Meyer. Phys. Rev. B 80, 012505 (2009).

[29] M.J. Hinton, S. Steers, B. Peters, F.Y. Yang, T.R. Lemberger. Phys. Rev. B 94, 014518 (2016).

[30] F. Bergeret, A.F. Volkov, K.B. Efetov. Phys. Rev. B 64, 134506 (2001).

[31] Y. Asano, A.A. Golubov, Y.V. Fominov, Y. Tanaka. Phys. Rev. Lett. 107, 087001 (2011).

[32] T. Yokoyama, Y. Tanaka, N. Nagaosa. Phys. Rev. Lett. 106, 246601 (2011).

[33] N. Pompeo, K. Torokhtii, C. Cirillo, A.V. Samokhvalov, E.A. Ilyina, C. Attanasio, A.I. Buzdin, E. Silva. Phys. Rev. B 90, 064510 (2014).

[34] A.V. Samokhvalov, A.I. Buzdin. Phys. Rev. B 92, 054511 (2015).

[35] Д. Сан-Жам, Г. Сарма, Е. Томас. Сверхпроводимость второго рода. Мир, М. (1970). С. 198.

[36] M. Eschrig. Rep. Prog. Phys. 78, 10450 (2015).

[37] В.П. Минеев, К.В. Самохин. Введение в теорию необычной сверхпроводимости. МФТИ, М. (1998). 144 с.

[38] L. Usadel. Phys. Rev. Lett. 25, 507 (1970).

[39] A.I. Buzdin. Rev. Mod. Phys. 77, 935 (2005).

[40] F.S. Bergeret, A.F. Volkov, K.B. Efetov. Rev. Mod. Phys. 77, 1321 (2005).

[41] T. Champel, M. Eschrig. Phys. Rev. B 71, 220506 (2005).

[42] М.Ю. Куприянов, В.Ф. Лукичев. ЖЭТФ 94, 139 (1988).

[43] Ya.V. Fominov, N.M. Chtchelkatchev, A.A. Golubov. Phys. Rev. B 66, 014507 (2002).

[44] А.А. Голубов, М.Ю. Куприянов, В.Ф. Лукичев, А.А. Орликовский. Микроэлектроника 12, 355 (1983).

[45] A.I. Buzdin, H. Kachkachi. Phys. Lett. A 225, 341 (1997). 